\title{
Harnessing structural instability and material instability in the hydrogel-actuated integrated responsive structures (HAIRS)
}

Yuhang $\mathrm{Hu}^{* \mathrm{a}}$, Philseok Kim ${ }^{\mathrm{b}}$ and Joanna Aizenberg ${ }^{\text {cdef }}$

a. Department of Mechanical Science and Engineering, University of Illinois at UrbanaChampaign, Urbana, Illinois 61801, USA.

b. SLIPS Technology Inc., 85 Bolton Street, Suite 122, Cambridge, Massachusetts 02140, USA.

c. John A. Paulson School of Engineering and Applied Sciences, Harvard University, Cambridge, Massachusetts 02138, USA.

d. Wyss Institute for Biologically Inspired Engineering, Harvard University, Cambridge, Massachusetts 02138, USA.

e. Department of Chemistry and Chemical Biology, Harvard University, Cambridge, Massachusetts 02138, USA.

f. Kavli Institute for Bionano Science and Technology, Harvard University, Cambridge, Massachusetts 02138, USA

* Corresponding E-mail: yuhanghu@illinois.edu 


\section{ABSTRACT}

We describe the behavior of a temperature-responsive hydrogel actuated integrated responsive structure (HAIRS). The structure is constructed by embedding a rigid high-aspect-ratio post in a layer of poly(N-isopropylacrylamide) hydrogel which is bonded to a rigid substrate. As the hydrogel contracts, the post abruptly tilts. The HAIRS has demonstrated its broad applications in generating reversible micro-pattern formation, active optics, tunable wettability, and artificial homeostasis. To quantitatively describe and predict the system behavior, we constructed an analytical model combining the structural instability, i.e. buckling of the post, and the material instability, i.e. the volume phase transition of PNIPAM hydrogel. The two instabilities of the system result in large hysteresis in response to heating and cooling processes. Experimental results validate the predicted phenomenon of the abrupt tilting as temperature and large hysteresis in a heating-and-cooling cycle in the PNIPAM actuated HAIRS. Based on this model, we further discuss the influence of the material properties on the actuation of the structure. 


\section{Introduction}

A network of crosslinked hydrophilic polymer chains can aggregate with water to form an elastomeric hydrogel. Hydrogels can be made responsive to external stimuli such as temperature [1], electric field [2], light [3], and $\mathrm{pH}$ [4]. Because of their stimuli-responsive properties, gels are used in diverse applications [5] such as drug delivery [6,7], cellular and tissue engineering [8], microfluidic devices [9,10], actuators and sensors [11,12]. Since most gels are soft and brittle, to improve their actuation force and mechanical integrity, hydrogels have been integrated with stiff materials and structures to form hybrid systems. A particular system of interest is the hydrogel actuated integrated response structure (HAIRS), in which an array of rigid high-aspect-ratio posts are embedded in a thin layer of hydrogel [13]. The contraction and expansion of the hydrogel in response to external stimuli drive the posts to bend and straighten up respectively. This hybrid system has been proved robust and utilized for various functions, such as reversible micro-pattern formation, active optics, tunable wettability and artificial homeostatic behavior [13-16]. For better understanding of the actuation mechanism and precise control in experiments, a theoretical model is in need.

In this paper, we describe a temperature-responsive HAIRS composed of an array of high-aspect-ratio posts and a chemically crosslinked poly(N-isopropylacrylamide) (PNIPAM) hydrogel. PNIPAM hydrogel is a temperature-responsive hydrogel with a lower critical solution temperature (LCST) of $32^{\circ} \mathrm{C}$. Below this temperature, the hydrogel is in swollen state but contracts when the temperature is above this value. The volume change of the PNIPAM hydrogel is discontinuous with respect to temperature, which is known as first-order volume-phase transition. While embedded in a layer of swollen PNIPAM hydrogel, the posts are in vertical position. As the hydrogel contracts, the posts are pulled down through the shear force built up 
along the interface between the posts and the hydrogel. To certain extent, the posts suddenly tilt from the vertical position, which is known as buckling instability. A theoretical model combining the material instability (i.e. the volume phase transition of PNIPAM gel) and structural instability (i.e. the buckling of the posts) is introduced to quantify the actuation of the HAIRS structure. The coupling of the two instabilities results in a hysteresis loop in response to heating and cooling processes. The tilting angle of the system is experimentally captured and compared with the theoretical model.

\section{Theoretical analysis}

\subsection{Homogeneous deformation}

An array of free-standing posts is embedded in a thin layer of PNIPAM hydrogel. The hydrogel layer is strongly bonded to both the rigid substrate and the posts (Fig. 1). When the structure is fabricated, the hydrogel is fully or partially swollen, and the post orients in vertical position. As the temperature increases, the hydrogel shrinks, and shear force is developed at the interface between the post and the hydrogel, which pulls the posts down. When the force is large enough, the posts suddenly tilt. When the temperature of the system decreases again, the hydrogel swells, bringing the posts back to the vertical position. In the case of an array of posts with the spacing between adjacent posts much smaller than the thickness of the gel layer as in previous studies [13-16], the deformation of the hydrogel layer can be essentially assumed to be homogeneous. To account for the full inhomogeneous deformation in the hydrogel, the solution of a detailed boundary value problem would be required. However, the result would be unlikely to alter the basic picture of the switching behavior of the structure. Therefore, to focus on the first-order behavior of the system and to facilitate understanding of the mechanism, we assume a homogeneous deformation in the analysis. 
As shown in Fig.1a, we take the dry network as the reference state and label every element with a coordinate $\left(X_{1}, X_{2}, X_{3}\right)$. When the hydrogel is formed and integrated with the post, there is certain amount of water in the pre-gel solution. The initial state of hydration of the hydrogel is denoted by a swelling ratio, $\lambda_{o}$, that can be calculated from the volume of the pre-gel solution, $V_{\text {gel }}$, and the volume of the dry monomers, $V_{\text {dry }}$, that is $V_{\text {gel }} / V_{\text {dry }}=\lambda_{0}^{3}$. The element of the dry network with label $\left(X_{1}, X_{2}, X_{3}\right)$ now is in a new position with coordinates $\left(\lambda_{0} X_{1}, \lambda_{0} X_{2}, \lambda_{0} X_{3}\right)$. When the hydrogel is in contracted state, the posts tilt. The tilting angle $\theta$ is denoted as the deviation from the vertical position (i.e. $\theta=0$ in vertical position). When the posts tilt, the elements of the network move to a new position with the current coordinates as [17]

$$
\begin{gathered}
x_{1}=\lambda_{0}\left(X_{1}+X_{3} \sin \theta\right), \\
x_{2}=\lambda_{0} X_{2}, \\
x_{3}=\lambda_{0} X_{3} \cos \theta .
\end{gathered}
$$

The function $x_{\mathrm{i}}\left(X_{1}, X_{2}, X_{3}\right)$ describes the field of deformation in the hydrogel in the tilting state. By definition, the deformation gradient $\mathbf{F}$ has the components $F_{\mathrm{ik}}=\partial x_{\mathrm{i}}\left(X_{1}, X_{2}, X_{3}\right) / \partial X_{\mathrm{K}}$. It is calculated from Eq. (1) as

$$
\mathbf{F}=\left[\begin{array}{ccc}
\lambda_{0} & 0 & \lambda_{0} \sin \theta \\
0 & \lambda_{0} & 0 \\
0 & 0 & \lambda_{0} \cos \theta
\end{array}\right]
$$

The volumetric swelling ratio of the hydrogel in the tilting state relative to its dry state is

$$
\operatorname{det}(\mathbf{F})=\lambda_{0}^{3} \cos \theta .
$$


This equation is readily understood. The thin layer of gel is bonded to the substrate, which constrains the in-plane deformation of the gel. The area of the gel layer remains $\lambda_{0}^{2}$ for a unit area of dry gel. What changes as the post tilts is the thickness of the gel layer, which equals $\lambda_{0}$ per unit thickness of dry gel when the post is in vertical position, and becomes $\lambda_{0} \cos \theta$ when the posts are in a tilting position. Equation (3) shows that the tilting causes the hydrogel to shrink in volume and allows the hydrogel to release water.

\subsection{Material model for PNIPAM hydrogel}

The hydrogel and the environment together constitute a thermodynamic system. We assume that the structure is immersed in a reservoir of water. The chemical potential of the water molecules in the reservoir is denoted as $\mu$. When the network imbibes a number of water molecules $C$, the free energy of the reservoir reduces by $\mu C$. Following Flory and Rehner [18], we assume that the free energy of the hydrogel consists of two parts: stretching energy due to the stretching of the polymer network and mixing energy due to the mixing between the polymers and water molecules. The free energy of the whole system becomes [19]

$$
G=W_{s}+W_{m}-\mu C,
$$

with the stretching energy being $[20,21]$

$$
W_{s}=\frac{1}{2} N k_{B} T\left[F_{i K} F_{i K}-3-2 \log (\operatorname{det} \mathbf{F})\right],
$$

and the mixing energy being [22]

$$
W_{m}=\frac{k_{B} T}{\Omega}\left[\Omega C \log \frac{\Omega C}{1+\Omega C}+\chi \frac{\Omega C}{1+\Omega C}\right],
$$


where $N$ defines the number of chains per unit volume of dry polymers, $k_{B} T$ the temperature in the unit of energy, $\Omega$ the volume per solvent molecule and $\chi$ a dimensionless measure of the strength of pairwise interactions between species.

We adopt the commonly used assumption that the change in the volume of the hydrogel equals the volume of water imbibed, namely [23]

$$
\Omega C=\operatorname{det}(\mathbf{F})-1 .
$$

Combining the above equations (2) to (7), we obtain an analytical expression for the free energy of the post-hydrogel system as

$$
\begin{aligned}
\frac{\Omega G(\theta)}{k_{B} T} & =\frac{1}{2} N \Omega\left[3\left(\lambda_{0}^{2}-1\right)-2 \log \left(\lambda_{0}^{3} \cos \theta\right)\right] \\
& +\left(\lambda_{0}^{3} \cos \theta-1\right)\left(\log \frac{\lambda_{0}^{3} \cos \theta-1}{\lambda_{0}^{3} \cos \theta}-\frac{\mu}{k_{B} T}\right), \\
& -\frac{\chi}{\lambda_{0}^{3} \cos \theta}
\end{aligned}
$$

where $\lambda_{0}$ is the initial swelling ratio of the PNIPAM hydrogel as it is fabricated, which is calculated from the composition of the amount of water and monomers in the precursor solution. The system has one degree of freedom, the tilting angle $\theta$.

\section{Experimental methods}

$N$-isopropylacrylamide (NIPAM) was purchased from Aldrich and purified by recrystallization from hexane. Dipentaerythritol pentaacrylate (SR-399) was kindly provided by Sartomer. All other chemicals were purchased from Aldrich and used as received.

Temperature-responsive hydrogel precursor stock solution was prepared in two parts. Solution A was prepared by dissolving $400 \mathrm{mg}$ of purified NIPAM, and 10/20 mg of $N, N-$ 
bis(dimethyl)acrylamide (BIS) as a crosslinker in $4 \mathrm{~mL}$ of deionized water. Solution B was prepared by dissolving $40 \mathrm{mg}$ of ammonium persulfate (APS) as a thermal radical initiator in 2 $\mathrm{mL}$ of deionized water. The two stock solutions were firstly cooled on an ice bath and mixed immediately before use. The hydrogels were fabricated by mixing $500 \mu \mathrm{L}$ of solution A and 250 $\mu \mathrm{L}$ of solution B with $5 \mu \mathrm{L}$ of catalyst $N, N, N^{\prime}, N^{\prime}$,-tetramethylethylenediamine (TEMED). Gelation took less than $5 \mathrm{~min}$ at room temperature. Gels with different amount of crosslinkers (BIS) were prepared. We denote the gels made from solution A containing $10 \mathrm{mg}$ BIS as 'Bis10 ' gels, and those made from solution A containing $20 \mathrm{mg}$ BIS as 'Bis-20' gels.

To ensure a strong covalent bonding of the hydrogel to the silicon substrate, we employed a rapid layer-by-layer dendritic growth of hyperbranched polyamine/polyacrylate thin film on a silicon substrate formed by repeated Michael addition reaction [24]. Briefly, an RCAcleaned silicon wafer (ca. $2 \mathrm{~cm} \times 2 \mathrm{~cm}$ ) was first treated with 3-aminopropylsilane followed by alternated dipping in 10 wt. \% methanol solutions of SR-399 and tris(2-aminoethyl)amine at room temperature for $10 \mathrm{~min}$ for at least two cycles. Between each dipping, the substrate was rinsed with plenty of methanol to remove excess materials from the surface. The last step was always dipping in the SR-399 solution to impart double bonds on the surface of the silicon substrate to allow for grafting-through formation of hydrogel on the substrate.

Different from our previous work in fabricating the HAIRS which contains an array of posts, here we fabricated the structure by using a single rigid rod for experimental convenience. The height of the rod is much larger than the thickness of the hydrogel layer. In this way, we can have a clearer view and more accurate measurement of the tilting angle. A surface-modified silicon substrate was placed on a lab jack. About $1 \mathrm{~cm}$ long and thin rigid wires $(250 \mu \mathrm{m}$ diameter) were prepared by cutting a stranded tinned copper alloy electrical wire. A rigid rod 
was held vertically using an alligator clip above the silicon substrate. The wire was engaged to the silicon substrate by slowly raising the lab jack while monitoring the gap and the angles with a wireless USB digital microscope camera (Model GT600, Firefly). To the post and substrate junction, freshly prepared $150 \mu \mathrm{L}$ of cold hydrogel precursor solution was applied to cover the junction. As the temperature of the precursor solution rises to room temperature, thermally initiated radical polymerization of the hydrogel layer took place within 5 min forming a uniformly thin and circular (ca. $1.5 \mathrm{~cm}$ diameter) hydrogel layer surrounding the junction.

The single post HAIRS sample was submerged in deionized water contained in a $9 \mathrm{~cm} \times$ $9 \mathrm{~cm}$ square plastic Petri dish. A rod-shaped stainless steel sheath cartridge heater $(35 \mathrm{~W}, 1 / 8$ inch diameter, 2 inches long, Model CSS01235/120V, Omega Engineering) connected to a variable transformer and a K-type thermocouple were placed in the Petri dish. The heating rate and the temperature were controlled/monitored using a temperature controller with an active feedback loop. Still images were captured from the side of the HAIRS sample perpendicular to its tilt using a wireless USB digital microscope camera for each temperature point after waiting for at least $4 \mathrm{~h}$ to reach equilibrium. Tilting angles were measured using ImageJ software. At least three measurements were used to calculate the average tilting angle and the standard deviation.

\section{Results and Discussions}

The material model described in Eqs. (4)-(6) contains two material parameters: $\chi$ and $N \Omega$. The parameter $\chi$ describes the enthalpy of mixing. In general, it depends on solvent concentration and temperature. A simple form has been proposed as [25]

$$
\chi(T, \varphi)=\chi_{0}+\chi_{1} \varphi
$$


where $\chi_{0}=A_{0}+B_{0} T, \chi_{1}=A_{1}+B_{1} T$ and $\phi=1 /(1+\Omega C)$. Here $\phi$ is the volume fraction of the polymer in the hydrogel. For PNIPAM monomers, the parameters have been characterized experimentally by Afroze et al. with $A_{0}=-12.947, A_{1}=17.92$, and $B_{1}=-0.0569 \mathrm{~K}^{-1}$ [26]. The dependence of the parameter $\chi$ on temperature and polymer concentration is also applicable for lightly crosslinked hydrogel [27]. In the following analysis, we will use these parameters to analyze the experimental results.

The parameter $N \Omega$ is related to the shear modulus of the hydrogel and can be characterized by free-swelling measurement. We took a piece of the prepared PNIPAM hydrogel and submerged it in deionized water, to swell the hydrogel without constraint. In equilibrium state, the free energy of the free swollen PNIPAM hydrogel per unit volume of its dry polymers is calculated from Eqs. (4)-(7) as

$$
\begin{aligned}
\frac{\Omega W_{f}\left(V / V_{0}\right)}{k_{B} T}= & \frac{1}{2} N \Omega\left[3\left(V / V_{0}\right)^{2 / 3}-3-2 \log \left(V / V_{0}\right)\right] \\
& +\left[\begin{array}{l}
\left(V / V_{0}-1\right) \log \left(1-\frac{1}{V / V_{0}}\right) \\
+\chi_{0}\left(1-\frac{1}{V / V_{0}}\right)+\chi_{1}\left(\frac{1}{V / V_{0}}-\frac{1}{\left(V / V_{0}\right)^{2}}\right)
\end{array}\right),
\end{aligned}
$$

where $V_{o}$ is the volume of the dry PNIPAM network, $V$ is the current volume of the PNIPAM hydrogel and $V / V_{0}$ is the volumetric swelling ratio of the network. For a given value of $N \Omega=0.01$, the free energy of a free-swelling hydrogel can be calculated at different temperatures as shown in Fig. 2a. By searching global minimum in the free energy at every temperature, we obtain the equilibrium-swelling ratio of the PNIPAM hydrogel as a function of temperature. Fitting this theoretical curve with experimental data, we can back out the unknown parameter, $N \Omega$. In the experiment, the hydrogels were fabricated with two different crosslinking densities. Figure $2 b$ 
plots the experimentally measured swelling ratio of the Bis-10 hydrogel and Bis-20 hydrogel at different temperatures. Plotted together are the theoretical curves with fitting parameters $N \Omega=0.01$ for Bis- 10 gel and $N \Omega=0.03$ for Bis -20 gel.

We fabricated two types of hydrogel-actuated single-post structures using the gels with the same composition but different crosslinking densities (Bis-10 and Bis-20 gels). From the volume of the monomers $V_{\text {dry }}$ and the volume of the precursor solution $V_{\text {gel }}$, the initial swelling ratio of the hydrogels can be obtained, $\lambda_{0}=\left(V_{\mathrm{gel}} / V_{\mathrm{dry}}\right)^{1 / 3}=2.2$. Inserting the values of the parameters $N \Omega, \chi$ and $\lambda_{0}$ into Eq. (10), the Gibbs free energy of the HAIRS now becomes a function of a single variable, the tilt angle of the post, $\theta$. As shown in Fig. 3a, the free energy of the HAIRS made of Bis-10 gel is plotted as a function of the tilting angle at several temperatures. At low temperature (e.g. $\mathrm{T}=32{ }^{\circ} \mathrm{C}$ ), there is only one local minimum at zero tilting angle. At high temperature (e.g. $\mathrm{T}=36{ }^{0} \mathrm{C}$ ), there are two local minima in free energy corresponding to two symmetric angles. At a particular temperature, $\mathrm{T}=33.5^{\circ} \mathrm{C}$, there are three equal local minima in the free energy curve, which corresponds to a phase-coexistent state, and this particular temperature is called phase-transition temperature. Searching the global minima in free energy at every temperature, we plotted the red dashed line in Fig. 3b. As the temperature increases, the tilting angle discontinuously changes from vertical to a high angle.

It is also shown in Fig. 3a that there is a range of temperatures under which the free energy has local minima at both zero angle and a high tilting angle. It indicates a possible hysteresis in response to heating and cooling processes. As shown the energy landscape in Fig. $3 \mathrm{a}$, at a temperature slightly below the phase-transition temperature (e.g. $\mathrm{T}=33{ }^{\circ} \mathrm{C}$ ), the local minimum in free energy at zero tilting angle is smaller than the local minimum at high tilting angle. At a temperature slightly above the phase-transition temperature (e.g. $\mathrm{T}=34{ }^{\circ} \mathrm{C}$ ), the local 
minimum in free energy at zero tilting angle is larger than the local minimum at high tilting angle. The smaller minimum corresponds to a stable state of equilibrium, the larger minimum corresponds to a metastable state, and the maximum corresponds to an unstable state. The local minima and maxima in free energy can be obtained by setting $\partial G(\theta) / \partial \theta=0$. The corresponding angles are plotted as a function of temperature in Fig. $3 \mathrm{~b}$ (black line). In the heating process, the structure starts from zero tilting angle. When the temperature is below the phase-transition temperature, the lowest minimum in free energy is at zero tilting angle, so the post stays vertically. As temperature increases to slightly above the phase-transition temperature, although the free energy at zero angle has a smaller value than at high tilting angle, the post may stay in vertical position. For the system to jump from vertical position to tilting position, it needs to overcome an energy barrier. Before the system acquires enough energy to jump, the post stays vertically. Similarly, in the cooling process, the structure initiates from high tilting angle and stays at tilting state even when the temperature is below the transition temperature. The largest hysteresis loop is between the two temperatures, beyond which there is only one global minimum in free energy (Fig. 3b).

It is also interesting to compare the temperature-responsive behavior of the HAIRS with free-swelling gel itself and constraint-swelling gels. In the constraint-swelling case, we consider a thin layer of hydrogel covalently bonded to a rigid substrate. The hydrogel layer swells only in vertical direction. The free energy of the gel layer is expressed as 


$$
\begin{aligned}
\frac{\Omega W_{c}\left(V / V_{0}\right)}{k_{B} T}= & \frac{1}{2} N \Omega\left[2 \lambda_{0}^{2}+\left(\frac{V / V_{0}}{\lambda_{0}^{2}}\right)^{2}-3-2 \log \left(V / V_{0}\right)\right] \\
& +\left[\begin{array}{l}
\left(V / V_{0}-1\right) \log \left(1-\frac{1}{V / V_{0}}\right) \\
\left.+\chi_{0}\left(1-\frac{1}{V / V_{0}}\right)+\chi_{1}\left(\frac{1}{V / V_{0}}-\frac{1}{\left(V / V_{0}\right)^{2}}\right)\right],
\end{array}\right.
\end{aligned}
$$

where $V / V_{0}$ is the volumetric swelling ratio of the PNIPAM hydrogel. For the three types of geometries described in Eqs. (8), (10) and (11), we search for the local minima and maxima in free energies at every temperature. The corresponding volumetric swelling ratio $V / V_{0}$ is plotted in Fig. 4. The volumetric swelling of the hydrogel in HAIRS structure is related to the tilting angle as $V / V_{0}=\lambda_{0}{ }^{3} \cos \theta$. The material parameters used in the plot are $N \Omega=0.01$ and $\lambda_{0}=2.2$, the same as for Bis-10 gel. The largest possible hysteresis loop is also illustrated for each case. In the freeswelling case, the largest hysteresis in response to the heating and cooling processes is only between $1{ }^{\circ} \mathrm{C}$ gap, which is so small that the hysteresis of PNIPAM gel was not captured in experiment before. In the constraint-swelling case, the lateral constraint provided by the bonding between the hydrogel and the substrate raise the energy barrier for the system to change between swollen state and contracted state. The hysteresis in the constraint-swelling case is larger than that in the free-swelling case. In the HAIRS structure, the hydrogel layer is not only bonded to the substrate, but also bonded to the post. As temperature increases, the hydrogel tends to contract, and shear stresses are built between the post and the hydrogel, which resist the hydrogel from contraction. When the shear force reaches a critical value, the post undergoes a buckling instability and suddenly tilts to a high angle. The tilting of the post allows the hydrogel to decrease its volume and release water. The lateral constraint of the substrate and the resistance of the post for tilting significantly raises the energy barrier for the hydrogel to change between 
swollen state and contracted state. The PNIPAM integrated HAIRS structure combines structural instability as well as material instabilities, and thus presents a pronounced hysteresis behavior.

The phenomenon of hysteresis in the post-PNIPAM structure is also observed experimentally. Two samples were tested. One was fabricated with Bis-10 gel and the other with Bis-20 gel. Pictures taken at different stages through heating and cooling processes are collected in Fig. 5. The extracted tilting angle is plotted as a function of temperature. The experimental measurements on the same sample have been consistent. The data points shown in Fig. 5b were from at least 3 independent measurements on each sample. The error is less than 5\%. Plotted together is the largest hysteresis loop that is predicted by theory. It shows appreciable agreement between experiment and theory. Although considerable discrepancies exist in the quantitative comparison, the experimental results verify the predicted key feature from the model on the large hysteresis in a heating-and-cooling cycle. The deviation between experiment and theoretical results could be due to the following reasons: in the experiment, we assemble the HAIRS structure with a single post instead of an array of homogeneously distributed posts. The deformation of the hydrogel is a field variable, which decays along the radial direction from the center of the post. The assumption of uniform and homogeneous deformation in theory does not describe the full radial inhomogeneous field of deformation of the hydrogel. However, for a first order approximation, this model still captures the basic switching behavior of the structure. Another consideration is the bonding between the post and the hydrogel. Any slippage in between will release the driving force for the post to tilt, which could be the reason that the experimentally measured tilt angle is smaller than theoretically predicted values.

The performance of the HAIRS can be tailored by controlling two variables: the crosslink density of the hydrogel and the solvent concentration of the precursor solution. In figure 6a, we 
fix the corsslinker density and vary the initial swelling ratio. For a larger initial swelling ratio, the gel can release a larger amount of water at high temperature and the post tilts to a larger angle in the contracted state. It also shows that the hysteresis behavior of the system in response to heating and cooling processes is larger for a hydrogel of higher initial swelling ratio. In figure $6 \mathrm{~b}$, we fix the initial swelling ratio of the hydrogel and vary its crosslinking density. It shows that the higher the crosslinking density, the higher the temperature for the system to be actuated.

Besides temperature, changing the relative humidity of the environment can also actuate the system. As is shown in Eq. (8), the chemical potential of the environment is related to its relative humidity $R H$ as $\mu / k_{B} T=\log R H$. The free energy of the HAIRS is plotted against the tilting angle at several relative humidity in Fig. 7. It can be observed that as humidity gradually changes, the global minimum in the free energy curve gradually changes from zero angle to a higher tilting angle. There is no such state as in the temperature controlled HAIRS, that local minima coexist at different angles. Therefore, HAIRS undergo second-order phase transition in response to humidity, while exhibiting first-order phase transition in response to temperature.

\section{Conclusions}

Based on Flory-Rehner theory, a material model has been presented for PNIPAM hydrogels. With fully characterized material parameters, the switching behaviour of a temperature-responsive HAIRS has been quantitatively modelled, which is analogous to the phase transition of a first kind. Due to the coexistence of the two instabilities, i.e. buckling of the

post and the volume phase transition of the PNIPAM hydrogel, the temperature-responsive HAIRS show significant hysteresis in response to the heating and cooling processes. Experimental results show appreciable agreement with the theoretical predictions. The model in this work is built for the HAIRS geometry. However, the sharp transition and significant 
hysteresis is a general phenomenon for structures that combines material and structural instabilities in their actuation mechanisms. This study provides theoretical guidance for future design of materials and structures for rapid actuation and energy dissipation.

\section{Acknowledgements}

This work was supported by the US Air Force Office of Scientific Research Multidisciplinary University Research Initiative under Award FA9550-09-1-0669-DOD35CAP.

Dr. Yuhang $\mathrm{Hu}$ acknowledges the funds from National Science Foundation (Grant No. 1554326). 


\section{References}

[1]. T. Tanaka, Collapse of Gels and the Critical Endpoint, Phys. Rev. Lett. 40 (1978) 820823.

[2]. T. Tanaka, I. Nishio, S-. T. Sun, and S. Ueno-Nishio, Collapse of gels in an electric field Science, 218 (1982) 467-469.

[3]. Suzuki, and T. Tanaka, Phase transition in polymer gels induced by visible light, Nature 346 (1990) 345-347.

[4]. T. Tanaka, D. Fillmore, S-.T. Sun, I. Nishio, G. Swislow, A. Shah, Phase transitions in ionic gels, Phys. Rev. Lett. 45 (1980) 1636-1639.

[5]. X. Hou, Design, Fabrication, Properties and Applications of Smart and Advanced Materials, CRC PRESS, New York (2016).

[6]. B. Jeong, Y. H. Bae, D. S. Lee, and S. W. Kim, Biodegradable block copolymers as injectable drug-delivery systems, Nature 388 (1997) 860-862.

[7]. Y. Qiu, and K. Park, Environment-sensitive hydrogels for drug delivery, Adv. Drug Delivery Rev. 53 (2001) 321-339.

[8]. C. Jen, M. C. Wake, and A. G. Mikos, Review: Hydrogels for cell immobilization, Biotechnol. Bioeng. 50 (1996) 357-364.

[9]. D. J. Beebe, J. S. Moore, J. M. Bauer, Q. Yu, R. H. Liu, C. Devadoss, and B.-H. Jo, Functional hydrogel structures for autonomous flow control inside microfluidic channels, Nature 404 (2000) 588-590.

[10]. L. Dong, A. K. Agarwal, D. J. Beebe, and H. R. Jiang, Adaptive liquid microlenses activated by stimuli-responsive hydrogels, Nature 442 (2006) 551-554. 
[11]. Richter, G. Paschew, S. Klatt, J. Lienig, K.-F. Arndt, and H.-J. P. Adler, Review on hydrogel-based pH sensors and microsensors, Sensors 8 (2008) 561-581.

[12]. G. Gerlach, M. Guenther, J. Sorber, G. Subchaneck, K.-F. Arndt, and A. Richter, Chemical and $\mathrm{pH}$ Sensors based on the swelling behaviour of hydrogels, Sens. Actuat. B 111-112 (2005) 555-561.

[13]. Sidorenko, T. Krupenkin, A. Taylor, P. Fratzl, and J. Aizenberg, Reversible switching of hydrogel-actuated nanostructures into complex micropatterns, Science 315 (2007) 487490.

[14]. S.H. Kang, B. Pokroy, L. Mahadevan, and J. Aizenberg, Control of Shape and Size of Nanopillar Assembly by Adhesion-Mediated Elastocapillary Interaction, ACS Nano 4 (2010) 6323-6331.

[15]. P. Kim, L.D. Zarzar, X. He, A. Grinthal, and J. Aizenberg, Hydrogel-Actuated Integrated Responsive Systems (HAIRS): Moving towards Adaptive Materials, Curr. Opin. Solid State Mater. Sci. 15 (2011) 236-245.

[16]. X. He, M. Aizenberg, O. Kuksenok, L.D. Zarzar, A. Shastri, A.C. Balazs, and J. Aizenberg, Synthetic Homeostatic Materials with Chemo-Mechano-Chemical SelfRegulation, Nature, 487 (2012) 214-218.

[17]. W. Hong, X. Zhao, and Z. Suo, Drying-induced bifurcation in a hydrogel-actuated nanostructure, J. Appl. Phys. 104 (2008) 084905.

[18]. P. J. Flory, and J. Rehner, Statistical mechanics of cross-linked polymer networks II swelling, J. Chem. Phys. 11 (1943) 521-526.

[19]. P. J. Flory, Principles of Polymer Chemistry, Cornell University Press, Ithaca, NY, 1953.

[20]. P. J. Flory, Thermodynamics of high polymer solutions, J. Chem. Phys 10 (1942) 51-61. 
[21]. M. L. Huggins, Solutions of long chain compounds, J. Chem. Phys. 9 (1941) 440.

[22]. M. L. Huggins, Revised theory of high polymer solutions, J. Chem. Phys. 86 (1964) $3535-3540$.

[23]. W. Hong, X. Zhao, J. Zhou, and Z. Suo, A theory of coupled diffusion and large deformation in polymeric gels, J. Mech. Phys. Solids 56 (2008) 1779-1793.

[24]. X. Wang, Y. Zhou, J. Xu, and H. Chen, Signal-on electrochemiluminescence biosensors based on CdS-carbon nanotube nanocomposite for the sensitive detection of choline and acetylcholine. Adv. Mater. 19 (2009) 1444-1450.

[25]. K. Otake, H. Inomata, M. Konno, and S. Saito, Thermal analysis of the volume phase transition with N-isopropylacrylamide gels, Macromolecules 23 (1990) 283-289.

[26]. F. Afroze, E. Nies, and H. Berghmans, Phase transitions in the system poly(Nisopropylacrylamide)/water and swelling behaviour of the corresponding network, J. Mol. Struct. 554 (2000) 55-68.

[27]. S. Cai, and Z. Suo., Mechanics and chemical thermodynamics of phase transition in temperature-sensitive hydrogels, J. Mech. Phys. Solids 59 (2011) 2259-2278. 
(a) reference state

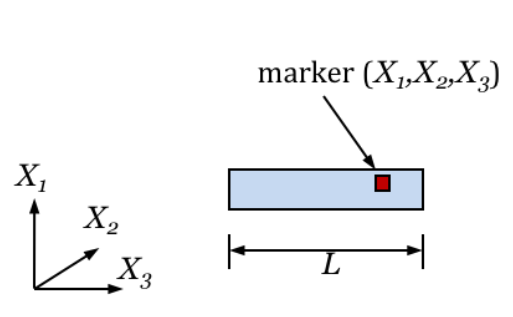

(b) fabrication state

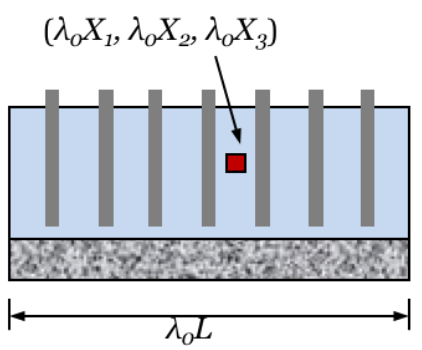

(c) actuation state

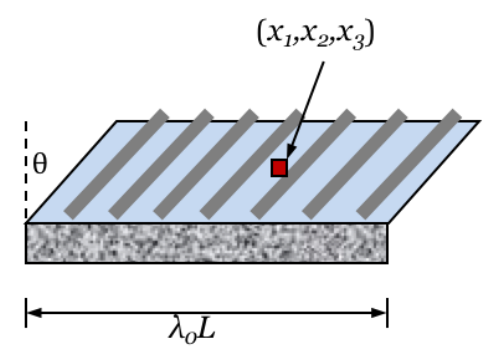

Fig. 1 A schematic illustration of the deformation of HAIRS with a material element coordinate specified in the reference, fabrication and tilting states 

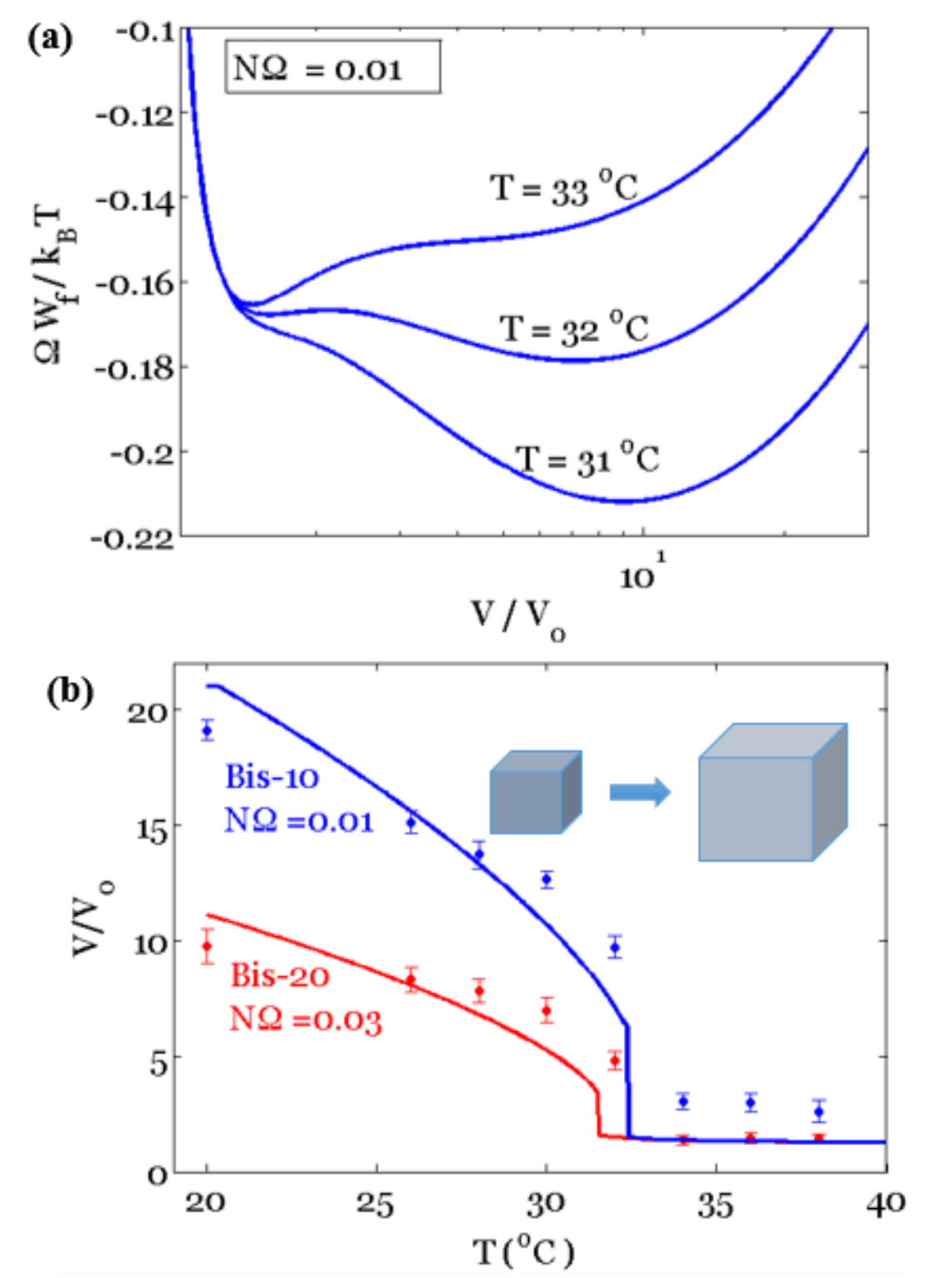

Fig. 2. (a) Free energy of a free-swelling PNIPAM hydrogel is plotted as a function of its volumetric swelling ratio at several temperatures. (b) The experimentally measured swelling ratios of the Bis-10 gels and Bis-20 gels are plotted in blue and red dots respectively. Together 
plotted solid lines are theoretical curves with fitting parameters $N \Omega=0.01$ (in blue) and $N \Omega=0.03$ (in red). 

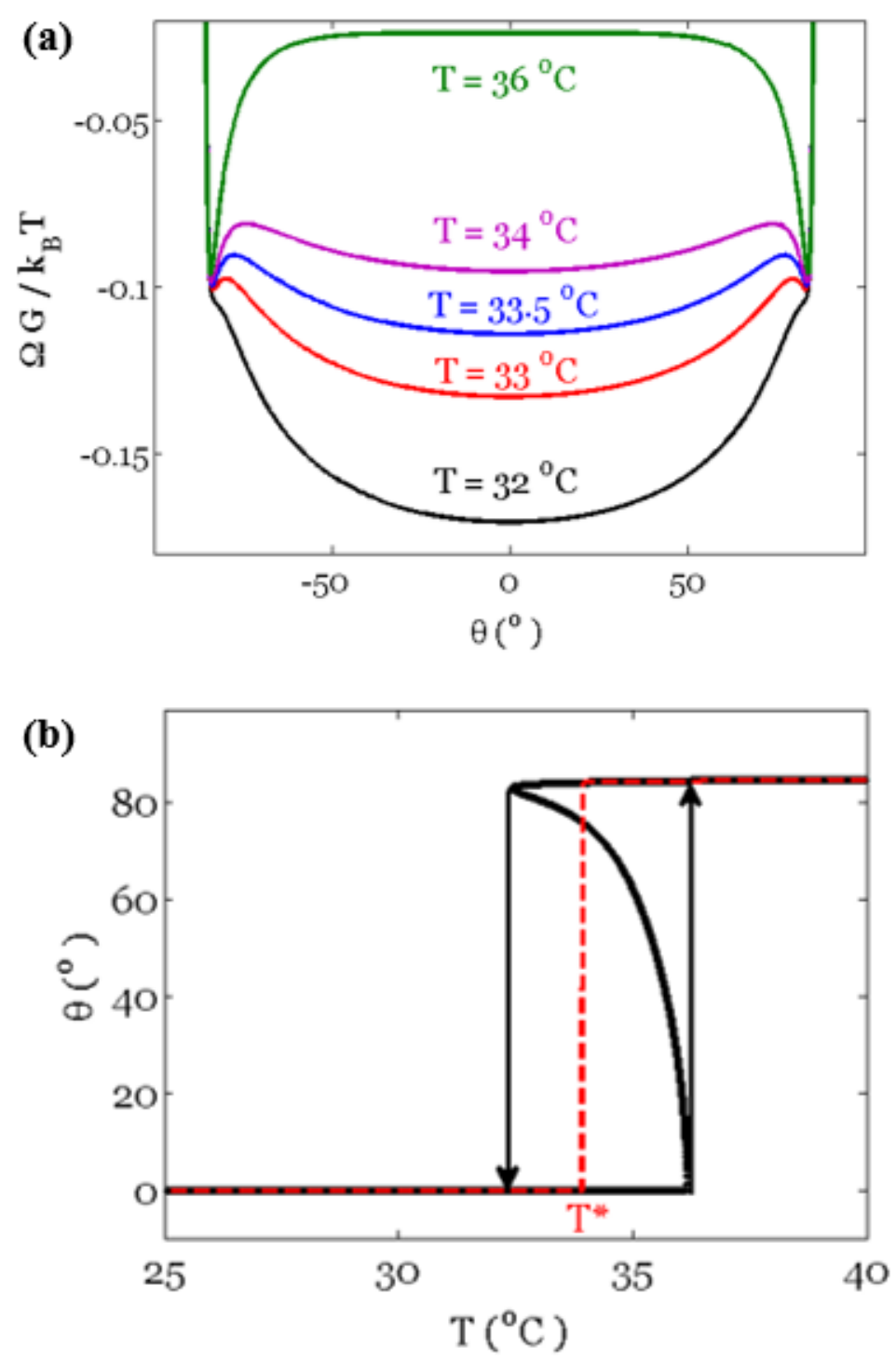

Fig. 3 (a) With given parameters $\lambda_{0}=2.2, N \Omega=0.01$, the Gibbs free energy of the temperature responsive HAIRS, is plotted against tilting angle at several temperatures. (b) The tilting angle, which minimizes the free energy of the HAIRS at every temperature, is plotted in red dashed line. The angle at which the free energy curve has either local minimum or maximum is collected 
at every temperature and plotted in solid black line. The Two arrows illustrate the theoretically largest hysteresis loop in response to heating and cooling processes. 


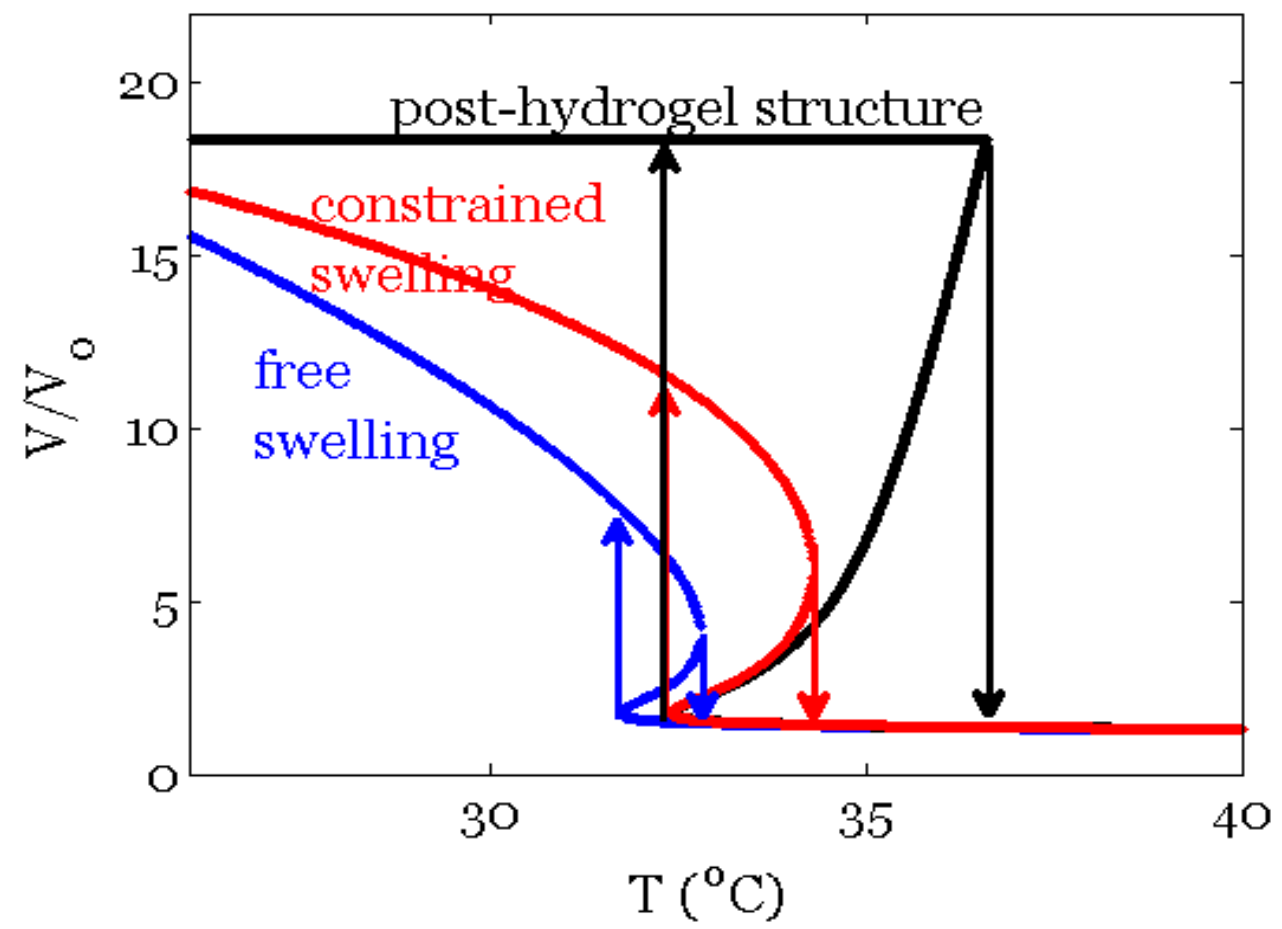

Fig. 4. The hysteresis behavior of a PNIPAM hydrogel in three geometries: a gel layer integrated in the HARIS (black line), a thin gel layer bonded to a rigid substrate (red line), and a free-swelling block (blue line). The volumetric swelling ratio of the PNIPAM hydrogel is plotted against temperature. The continuous curves are derived from searching every local minimum and maximum in free energy at every temperature. The two arrows in each curve illustrate the theoretically largest hysteresis loop in response to heating and cooling processes. 
(a)

heating

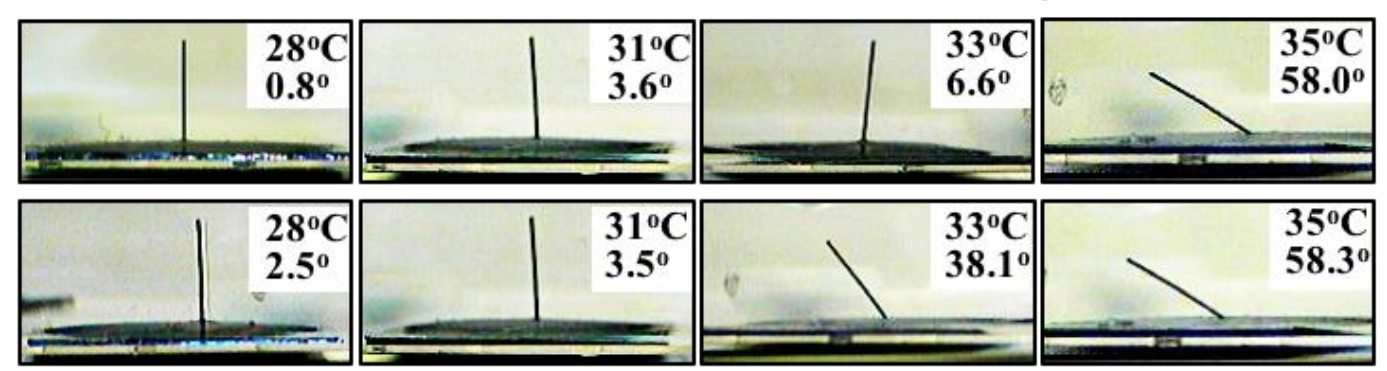

cooling

(b)

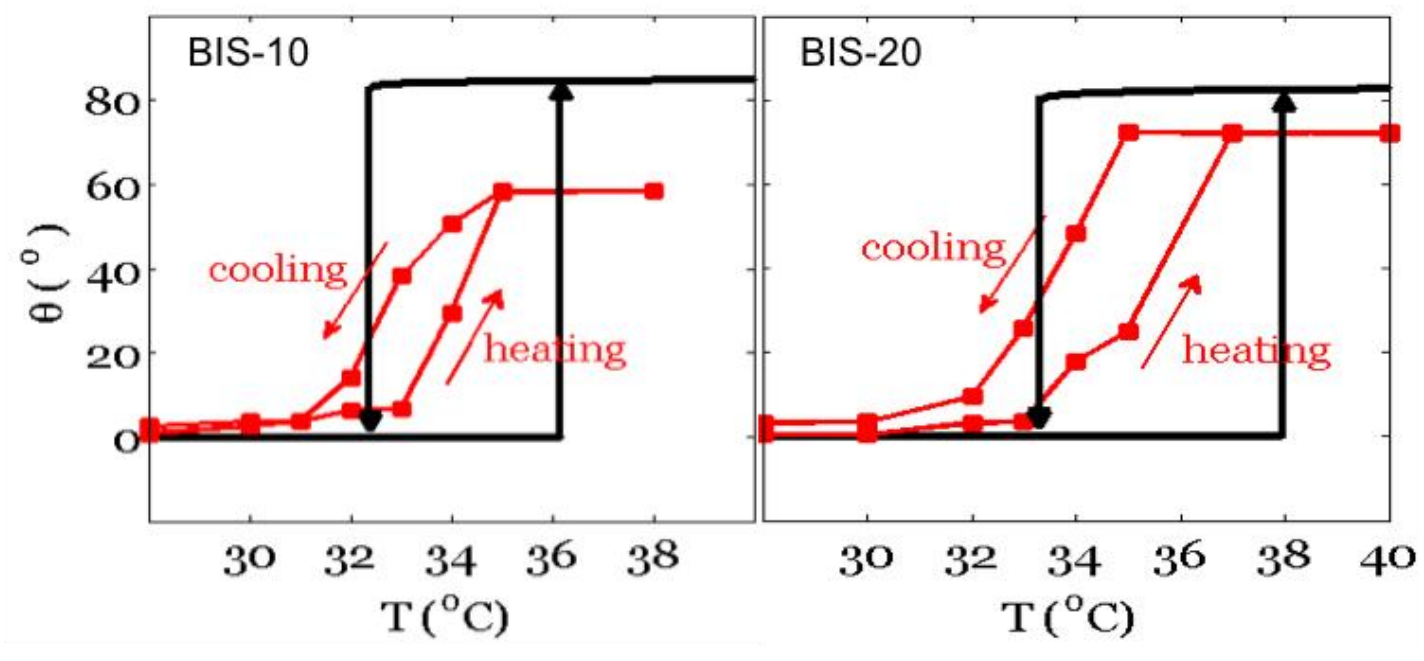

Fig. 5 (a) Pictures of the post actuated by Bis-10 gel over one heating and cooling cycle. (b) The experimental tilting angles captured from both Bis-10 and Bis-20 integrated structures are plotted as a function of temperature in red dots. The theoretically largest hysteresis loop is plotted in black line. 


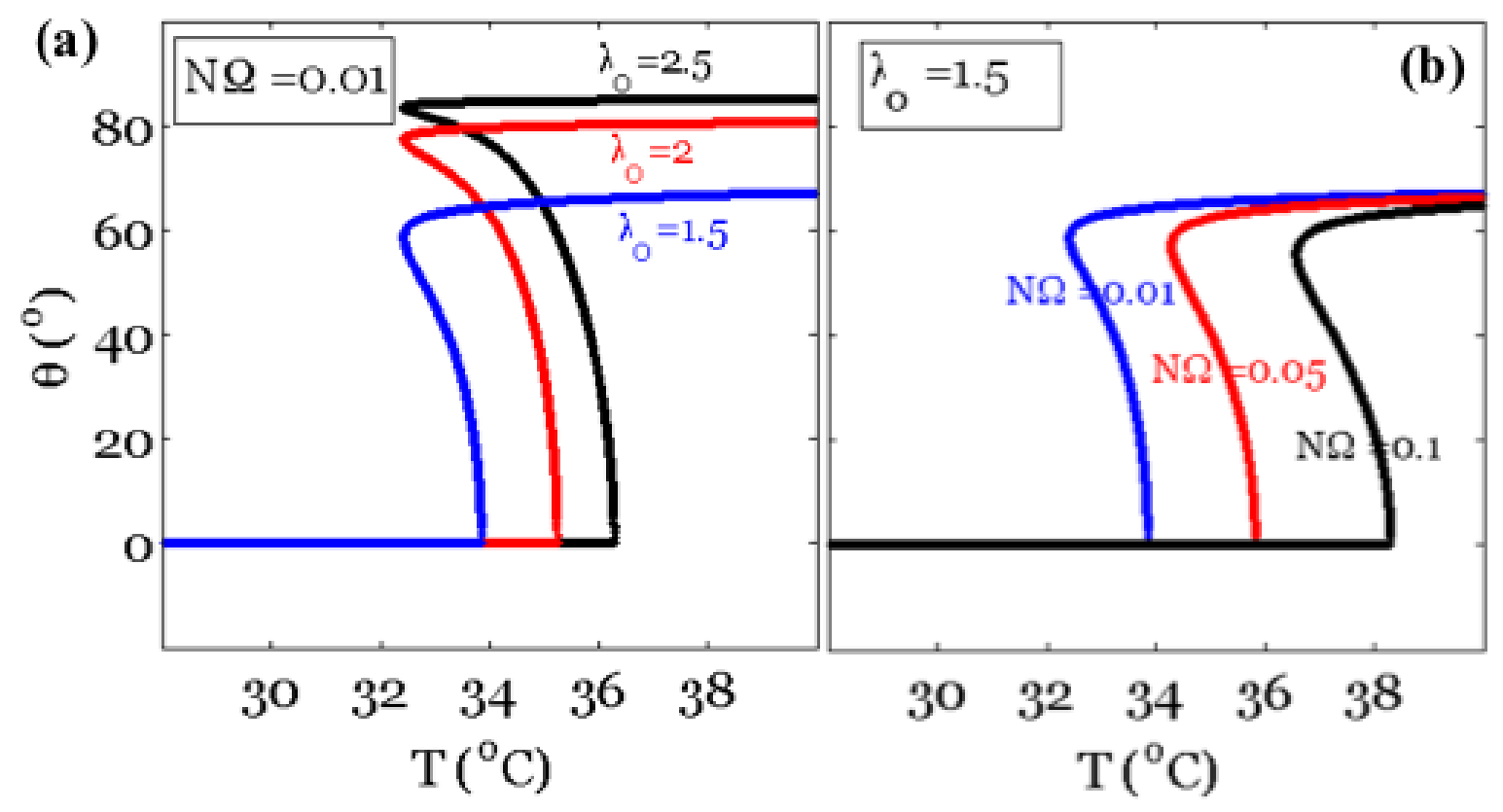

Fig. 6 The tilting angle is plotted as temperature (a) with fixed crosslink density $N \Omega=0.01$ but varying initial swelling ratios, and (b) with fixed initial swelling ratio $\lambda_{0}=1.5$ but varying crosslink densities. 


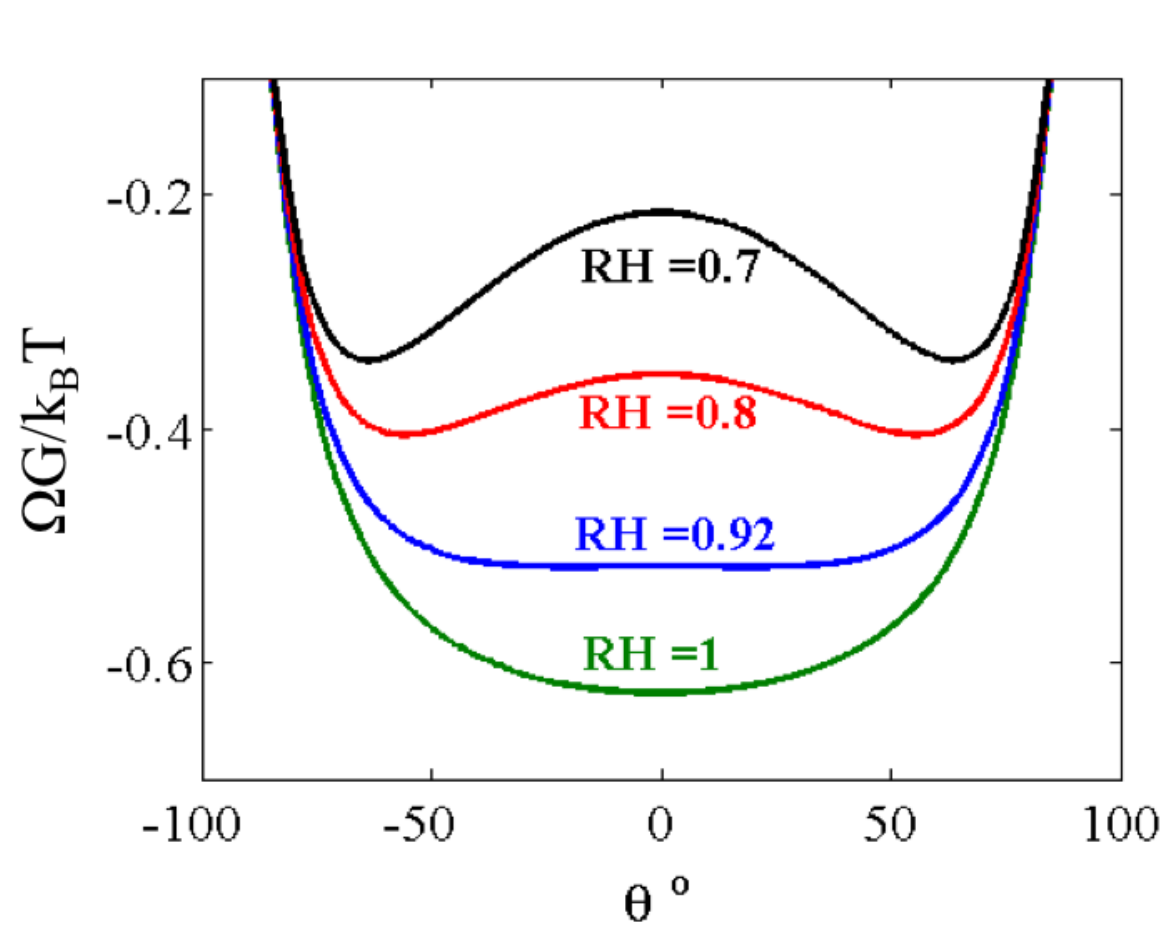

Fig. 7 The free energy of the HAIRS is plotted against the tilting angle at several values of the relative humidity. 